\title{
Radiation driven instability of rapidly rotating relativistic stars: criterion and evolution equations via multipolar expansion of gravitational waves
}

\author{
A. I. Chugunov \\ Ioffe Institute, St Petersburg, Russia.
}

\begin{abstract}
I suggest a novel approach for deriving evolution equations for rapidly rotating relativistic stars affected by radiation-driven Chandrasekhar-Friedman-Schutz (CFS) instability. This approach is based on the multipolar expansion of gravitational wave emission and appeals to the global physical properties of the star (energy, angular momentum, and thermal state), but not to canonical energy and angular momentum, which is traditional. It leads to simple derivation of the CFS instability criterion for normal modes and the evolution equations for a star, affected by this instability. The approach also gives a precise form to simple explanation of the CFS instability: it occurs when two conditions met: (a) gravitational wave emission removes angular momentum from the rotating star (thus releasing the rotation energy) and (b) gravitational waves carry less energy, than the released amount of the rotation energy. To illustrate the results I take the r-mode instability in slowly rotating Newtonian stellar models as an example. It leads to evolution equations, where the emission of gravitational waves directly affects the spin frequency, being in line with the arguments by Levin \& Ushomirsky (2001b), but in contrast to widely accepted equations by Lindblom, Owen, \& Morsink (1998); Ho \& Lai (2000). According to the latter, effective spin frequency decrease is coupled with dissipation of unstable mode, but not with the instability as it is. This problem, initially stressed by Levin \& Ushomirsky (2001b), is shown to be superficial, and arises as a result of specific definition of the effective spin frequency, applied by Lindblom et al. (1998); Ho \& Lai (2000). Namely, it is shown, that if this definition is taken into account properly, the evolution equations coincide with obtained here in the leading order in mode amplitude. I also argue that the next-to-leading order terms in evolution equations (which differ for Owen et al. 1998 and Ho \& Lai 2000) require clarification and thus it would be more self-consistent to omit them.
\end{abstract}

Keywords: stars: neutron; instabilities; gravitational waves

\section{Introduction}

Andersson (1998); Friedman \& Morsink (1998) demonstrate that all rotating stars are unstable with respect to excitation of r-modes (similar to Earths Rossby waves controlled by the Coriolis force) at any rotation rate, if dissipation is neglected. It is a particular case of Chandrasekhar-Friedman-Schutz (CFS) instability (Chandrasekhar 1970; Friedman \& Schutz 1978a,b). Evolution equations for dissipative neutron star, affected by the r-mode instability, were derived by Owen et al. (1998) (see also Levin 1999; Ho \& Lai 2000; Alford \& Schwenzer 2014a; Gusakov et al. 2014a), assuming slow rotating Newtonian stellar model. These equations are widely applied in literature and many observational consequences were predicted (see Haskell 2015; Chugunov et al. 2017, for recent reviews). In par- ticular, the r-mode instability can limit spin frequencies of neutron stars (Bildsten 1998; Andersson et al. 1999), generate potentially observable gravitational waves (e.g. Owen 2010), and lead to anti-glitches in millisecond pulsars and neutron star, accreting in low mass X-ray binaries (Kantor, Gusakov, \& Chugunov 2016) and even formation of additional class of hot rapidly rotating neutron stars - HOFNARS, - which could reveal itself by stable thermal emission from surface, but do not accrete (Chugunov, Gusakov, \& Kantor 2014). Confronting observations with predictions of r-mode instability theory one can put important constraints on the physics of neutron stars, including properties of their depths (see e.g., Haskell, Degenaar, \& Ho 2012; Haskell 2015; Chugunov et al. 2017).

To a great extent the above results are based on the canonical energy formalism and Lagrangian perturba- 
tion theory formulated by Friedman \& Schutz (1978a,b) and generalized for relativistic case by Friedman (1978). This theory is, of course, mathematically strict, but it is but rather complicated: Friedman \& Schutz (1978a,b) reveal class of Lagrangian displacements, - called trivials, - which do not modify physical variables and introduce another class of Lagrangian displacements - called of canonical, - which are orthogonal to the trivials. They also introduce canonical energy functional for Lagrangian perturbations and demonstrate that perturbations described by canonical Lagrangian displacement with negative canonical energy are unstable with respect to gravitational radiation (in absence of viscosity). It is worth to note that canonical energy can be equal to the physical change of energy under certain conditions (e.g., growth of normal mode under action of radiation reaction force in absence of viscosity), but generally it is not a case (Friedman \& Schutz 1978a,b). This feature leads to conceptual critique (Levin \& Ushomirsky 2001b, see also footnote 2) of the first derivation of evolution equations for CFS unstable star (Owen et al. 1998). Objections were rather convincingly replied to by Ho \& Lai (2000), who suggest slightly modified evolution equations. Currently both versions are applied by different authors (e.g., compare Haskell 2015 and Mahmoodifar \& Strohmayer 2013), but which of them is more precise?

To see the problem in a different light, I suggest another approach, which as I believe, is also useful from methodological point of view. Namely, I avoid to use Lagrangian perturbation theory and canonical energy and angular momentum of perturbations, but deal with global physical properties of the star (energy, angular momentum, and the thermal state) and consider instability of normal modes. ${ }^{1}$ The key point is Eq. (3), which follows from the multipolar expansion of gravitational wave emission (Thorne 1980) and couples the rates of change of the energy and the angular momentum. It naturally reveals physics of CFS instability: emission of gravitational waves should remove angular momentum from the star, thus releasing the rotation energy. The instability occurs if gravitational waves carry less energy than the released amount of the rotation energy (see first equality in Eq. 4).

Similar explanation was given, e.g., by Andersson (1998); Andersson \& Kokkotas (2001), but that was rather heuristic arguments than strict proof (e.g., Friedman \& Stergioulas 2014). ${ }^{2}$ Explicit reference to Eq. (3) allows to formulate these arguments in a pre-

\footnotetext{
${ }^{1}$ It is worth to note, that Lagrangian perturbation theory developed by Friedman \& Schutz (1978a,b); Friedman (1978) allows to consider instability of the initial data with no assumptions concerning the existence or completeness of normal modes.

${ }^{2}$ Let me note that earlier it was typically assumed that unstable perturbation (as it is) decreases physical angular momentum. However, as noted by Levin \& Ushomirsky (2001b), it is rather misleading, and, for example, such perturbations can exist in a
}

cise form and not only derive instability criterion, but also obtain general evolution equations for CFS unstable star (see Sec. 2). It is worth noting, that this derivation does not require simplifying assumptions of slow rotation or Newtonian gravitation, but is valid in full general relativity framework. Additional advantage of derivation in Sec. 2 is that it allows straightforward generalization for superfluid neutron stars, because it exploits the global properties of the star (energy and angular momentum), but not complicated structure of internal perturbations.

To apply the evolution equations, derived in Sec. 2 one, of course, should deal with detailed description of internal perturbations to calculate efficiency of the gravitational radiation and dissipation timescales. It is very complicated problem, which can be crucially affected by the neutron star core composition (e.g., Jones 2001; Lindblom \& Owen 2002; Nayyar \& Owen 2006; Alford \& Schwenzer 2014b), superfluidity (Yoshida \& Lee 2003a; Lee \& Yoshida 2003; Andersson et al. 2009; Haskell et al. 2009; Gusakov et al. 2014b; Kantor \& Gusakov 2017), crustcore coupling (Rieutord 2001; Levin \& Ushomirsky 2001a; Glampedakis \& Andersson 2006a,b), in-medium effects (Kolomeitsev \& Voskresensky 2015), and, of course, general relativity (e.g., Kojima 1998; Lockitch, Andersson, \& Friedman 2001; Yoshida \& Lee 2002; Yoshida \& Lee 2003b; Ruoff \& Kokkotas 2002; $\quad$ Lockitch, Friedman, \& Andersson 2003; Lockitch, Andersson, \& Watts 2004; Krüger, Gaertig, \& Kokkotas 2010). However, I leave these problems beyond the scope of this paper, because they provide parameters for the evolution equations, but do not modify their form.

In section $3 \mathrm{I}$ illustrate the general evolution equations on the example of slow rotating Newtonian stellar model. In particular, it is shown that the gravitational radiation directly leads that the star spins down, being thus in agreement with arguments by Levin \& Ushomirsky (2001b). In section 4 these results are compared with widely applied equations by Owen et al. (1998); Ho \& Lai (2000). At the first glance my equations differ from those in both of papers: latter attribute decrease of the effective spin frequency (as introduced by Owen et al. 1998; Ho \& Lai 2000) to the dissipation of the unstable mode, but not with instability as it is. I demonstrate that this difference is associated with definition of the effective frequency and just as it is taken into account, the evolution equations agree in the leading order in mode amplitude. I also argue that the next-to-leading order terms, which differ for Owen et al. (1998) and Ho \& Lai (2000), was not yet

star with same physical angular momentum as unperturbed configuration (see also thorough discussion of the 'wave-momentum' myth by McIntyre 1981). 
derived accurately and should be omitted. I conclude in section 5 .

\section{CFS instability and evolution of unstable star}

In this section I consider rotating relativistic star in its asymptotic rest frame; their total mass-energy $E$ and angular momentum $J$ are well defined (see, e.g., Sec. 19 in the textbook by Misner, Thorne, \& Wheeler 1973). For given angular momentum $J$, uniform rotation corresponds to the minimal energy (at fixed baryon number), which is the rotational energy $E_{\text {rot }}$ (e.g. Boyer \& Lindquist 1966; Hartle \& Sharp 1967; Stergioulas 2003). Corresponding spin frequency is (it follows, e.g., from variational principle by Hartle \& Sharp 1967)

$$
\Omega=\frac{\partial E_{\mathrm{rot}}}{\partial J} .
$$

If star is perturbed, but the total angular momentum does not changed, the energy $E$ exceeds rotational en$\operatorname{ergy}\left(E>E_{\text {rot }}\right)$, leading to positively defined excitation energy:

$$
E_{\mathrm{ex}}=E-E_{\mathrm{rot}} .
$$

I would like to stress, that $E_{\text {ex }}$ should not be confused with non-positively-defined canonical energy, introduced by Friedman \& Schutz (1978a,b).

As shown by Thorne (1980), the rate of changes of the energy $\dot{E}^{\mathrm{GR}}$ and angular momentum $\dot{J}^{\mathrm{GR}}$ due to emission of gravitational waves can be expressed as sums over multipolar contributions, which comes from expansion of radiation field in the local wave zones. As it follows from Eqs. (4.16) and (4.23) by Thorne (1980), for perturbations $\propto \mathrm{e}^{\imath(\omega t+m \phi)}$, these rates are coupled by the equation ${ }^{3}$

$$
-\frac{\omega}{m} \dot{J}^{\mathrm{GR}}=\dot{E}^{\mathrm{GR}} .
$$

Gravitational wave emission removes energy from the system, thus $\dot{E}^{\mathrm{GR}}<0$. Sign of rate of change of angular momentum is determined by $\omega / \mathrm{m}$. The rate of change of the excitation energy $E_{\text {ex }}$ is

$$
\dot{E}_{\mathrm{ex}}^{\mathrm{GR}}=\dot{E}^{\mathrm{GR}}-\dot{E}_{\mathrm{rot}}^{\mathrm{GR}}=\left(1+\frac{m \Omega}{\omega}\right) \dot{E}^{\mathrm{GR}} .
$$

Here $\dot{E}_{\mathrm{rot}}^{\mathrm{GR}}=\Omega \dot{J}^{\mathrm{GR}}$. The excitation energy is increased by emission of gravitational waves if and only if $(1+$ $m \Omega / \omega)<0$. This condition is equal to well known criterion of CFS instability (see e.g. Friedman \& Schutz 1978a; Friedman 1978; Andersson \& Kokkotas 2001; Friedman \& Stergioulas 2014): the prograde mode pattern in the inertial frame $(-\omega / m>0)$, but retrograde

\footnotetext{
${ }^{3}$ This equation is well known for electromagnetic waves, see e.g.
} Sec. 9.8 in textbook by Jackson (1999). mode pattern in the frame, corotating with the star $(-\Omega-\omega / m<0)$. Thus the above discussion proves the CFS instability criterion for normal modes without appeal to the Lagrangian perturbation theory.

To describe the evolution of CFS unstable star, I parametrize it state by three parameters: (i) total angular momentum $J$, (ii) the mode energy $E_{\text {ex }}$, and (iii) thermal state. The latter can be characterized by temperature in the stellar centre $T$, because neutron stars are almost isothermal (e.g., Page, Lattimer, Prakash, \& Steiner 2004; Gusakov, Kaminker, Yakovlev, \& Gnedin 2005) because of high thermal conductivity in their depths (see, e.g., Shternin, Baldo, \& Haensel 2013 for recent results).

Evolution of angular momentum due to emission of gravitational waves is described by Eq. (4)

$$
\dot{J}^{\mathrm{GR}}=-\frac{m}{\omega+m \Omega} \dot{E}_{\mathrm{ex}}^{\mathrm{GR}} .
$$

In this equation, which is applicable for any oscillation mode (in particular for r-modes) at any spin frequency and even for general relativistic (not Newtonian) stellar models, $\Omega=\Omega(J)$ is determined by Eq. (1).

Evolution of the mode energy is associated with energy pumping by gravitational waves $\dot{E}_{\mathrm{ex}}^{\mathrm{GR}}$ and energy losses due to dissipation $\dot{E}_{\mathrm{ex}}^{\text {dis }}$

$$
\dot{E}_{\mathrm{ex}}=\dot{E}_{\mathrm{ex}}^{\mathrm{GR}}+\dot{E}_{\mathrm{ex}}^{\mathrm{dis}} .
$$

Finally, the thermal evolution of star follows

$$
C \dot{T}=-\dot{E}_{\mathrm{ex}}^{\mathrm{dis}}-L_{\mathrm{cool}} .
$$

Here $L_{\text {cool }}$, and $C$ are total cooling power (neutrino and thermal emission from surface) and heat capacity of the star respectively. One can also add torques, which are not associated with r-modes (e.g., accretion spin up, e.g., Ghosh \& Lamb 1979; Wang 1995 or magnetic braking, e.g., Beskin, Gurevich, \& Istomin $1993)$ to the angular momentum evolution equation (5) and additional heating [e.g., accretion-induced deep crustal heating (Brown, Bildsten, \& Rutledge 1998) or internal heating in millisecond pulsars (Alpar, Pines, Anderson, \& Shaham 1984; Reisenegger 1995; Gusakov, Kantor, \& Reisenegger 2015)] to the thermal evolution equation (7).

To apply equations (5)-(7) one should specify properties of the mode: $\omega, \dot{E}_{\mathrm{ex}}^{\mathrm{GR}}$ and $\dot{E}_{\mathrm{ex}}^{\mathrm{dis}}$ as function of $E_{\text {ex }}$ and $J$, which is, of course, very complicated problem, especially for relativistic stellar models (see e.g. Lockitch et al. 2003; Kastaun 2011). However, for slow rotating Newtonian stellar models these parameters can be easily extracted from literature (yet, depending on the microphysical assumptions, see e.g., Haskell 2015) and in the next section they are applied to illustrate equations (5)-(7). 


\section{Evolution of r-mode unstable neutron star within slow rotating Newtonian stellar models}

Here I restrict myself to slow rotating Newtonian stellar models, which are commonly used to study CFS instability in neutron stars (see, e.g., Haskell 2015 for recent review). In this case, Eq. (1) can be written as $\Omega=J / I$, where moment of inertia $I$ does not depend on $J$. The most unstable mode is r-mode with $l=m=2$ (e.g., Lindblom et al. 1998), with the frequency

$$
\omega=-\frac{(m-1)(m+2)}{m+1} \Omega=-\frac{4}{3} \Omega
$$

The first order Eulerian perturbations of velocity can be written as follows (e.g., Provost, Berthomieu, \& Rocca 1981):

$$
\delta^{(1)} \boldsymbol{v}=\alpha R \Omega\left(\frac{r}{R}\right)^{m} \boldsymbol{Y}_{m m}^{B} \exp ^{i \omega t}
$$

Here $\alpha$ is dimensionless mode amplitude and

$$
\boldsymbol{Y}_{l m}^{B}=\frac{1}{l(l+1)} r \nabla \times\left(r \nabla Y_{l m}\right)
$$

is magnetic-type vector spherical harmonic (see e.g., Varshalovich, Moskalev, \& Khersonskii 1988). The excitation energy for r-mode can be written in the form

$$
\begin{aligned}
E_{\mathrm{ex}} & =\int \frac{\rho \delta v^{2}}{2} \mathrm{~d}^{3} \boldsymbol{r}=\int \frac{\rho\left[\delta^{(1)} \boldsymbol{v}\right]^{2}}{2} \mathrm{~d}^{3} \boldsymbol{r} \\
& +\int \rho \boldsymbol{v}_{0} \delta^{(2)} \boldsymbol{v} \mathrm{d}^{3} \boldsymbol{r}+\mathcal{O}\left(\alpha^{3}\right)
\end{aligned}
$$

Here $\delta v^{2}=(\boldsymbol{v})^{2}-\left(\boldsymbol{v}_{0}\right)^{2}=2 \boldsymbol{v}_{0} \delta \boldsymbol{v}+(\delta \boldsymbol{v})^{2}$ is perturbation of squared velocity and $\delta \boldsymbol{v}=\sum_{i} \delta^{(i)} \boldsymbol{v}$ is total perturbation of velocity, presented as a sum over orders in $\alpha$ [i.e., $\delta^{(i)} \boldsymbol{v}=\mathcal{O}\left(\alpha^{i}\right)$ ]. The integral is taken over stellar volume. I neglect also density pertrubations, because they are of the second order in $\Omega$ (e.g., Lindblom et al. 1998). The second term in Eq. (11) depend on the second order velocity perturbation $\delta^{(2)} \boldsymbol{v}$, but because of the finite velocity at the unperturbed state $\boldsymbol{v}_{0}$, it contributes to the energy at the same order as first order perturbations (Friedman \& Schutz 1978a). ${ }^{4}$ However, only axysimetric part $\delta_{\mathrm{sym}}^{(2)} \boldsymbol{v}$ can contribute to the integral, ${ }^{5}$ but the definition of excitation energy (Eq. 2) supposes that the perturbed state has the same angular momentum as unperturbed star, constraining $\delta \boldsymbol{v}$ :

$$
\delta J=\int \rho[\delta \boldsymbol{v} \times \boldsymbol{r}] \mathrm{d}^{3} \boldsymbol{r}=0 .
$$

\footnotetext{
${ }^{4}$ The linear contribution from the first order perturbation vanished after integration over stellar volume, e.g., Levin \& Ushomirsky (2001b).

${ }^{5}$ For given first order solution, the asymmetric part $\delta_{\mathrm{sym}}^{(2)} \boldsymbol{v}$ is determined up to arbitrary cylindrically stratified differential rotation (e.g., Sá 2004).
}

pasa (2018)

doi:10.1017/pas.2018.xxx
As far as unperturbed state is uniform rotation $\boldsymbol{v}_{0}=$ $\boldsymbol{\Omega} \times \boldsymbol{r}$, the contribution of $\delta_{\mathrm{sym}}^{(2)} \boldsymbol{v}$ to the energy should vanish in Eq. (11) at the second order in $\alpha$. Thus, the second order excitation energy is determined exclusively by the first order perturbations and equals to the kinetic energy in the system corotating with star. It can be written as follows (see, e.g., Lindblom et al. 1998):

$$
E_{\text {ex }}=\frac{1}{2} \alpha^{2} \Omega^{2} R^{-2 m+2} \int_{0}^{R} \rho r^{2 m+2} \mathrm{~d}^{3} \boldsymbol{r}
$$

The instability timescale

$$
\tau^{\mathrm{GR}}=-2 \frac{E_{\mathrm{ex}}}{\dot{E}_{\mathrm{ex}}^{\mathrm{GR}}}
$$

can be calculated via multipolar expansion of gravitational radiation for Newtonian sources (see Sec. V.C in Thorne 1980), as it was done by Lindblom et al. (1998):

$$
\begin{aligned}
\frac{1}{\tau^{\mathrm{GR}}} & =-\frac{32 \pi G \Omega^{2 m+2}}{c^{2 m+3}} \frac{(m-1)^{2 m}}{[(2 m+1) ! !]^{2}}\left(\frac{m+2}{m+1}\right)^{2 l+2} \\
& \times \int_{0}^{R} \rho r^{2 m+2} \mathrm{~d} r
\end{aligned}
$$

This result agrees with analytic treatment of r-mode instability up to second order in oscillation amplitude by Friedman et al. (2016).

The dissipation rate

$$
\tau^{\mathrm{dis}}=-2 \frac{E_{\mathrm{ex}}}{\dot{E}_{\mathrm{ex}}^{\mathrm{dis}}}
$$

should be specified for each certain model of dissipation (shear viscosity, mutual friction, etc.). Note, internal dissipative processes can not affect total angular momentum of the star, thus the rotational energy is conserved and dissipation time scale can be estimated from dissipation rate of the total energy $\dot{E}^{\text {dis }}$ (i.e. one can substitute $\dot{E}^{\text {dis }}$ instead of $\dot{E}_{\mathrm{ex}}^{\text {dis }}$ in Eq. 16). For example, the contribution of the shear viscosity $\eta$ to the dissipation rate is (Lindblom et al. 1998):

$$
\tau^{\mathrm{S}}=(m-1)(2 m+1) \frac{\int_{0}^{R} \eta r^{2 l} \mathrm{~d} r}{\int_{0}^{R} \rho r^{2 l+2} \mathrm{~d} r} .
$$

Introduction of these timescales gives $\dot{E}_{\mathrm{ex}}^{\mathrm{GR}}$, and $\dot{E}_{\mathrm{ex}}^{\mathrm{dis}}$ as functions of $J$ and $E_{\text {ex }}$, allowing thus to rewrite Eqs. (5) - (7) in the form:

$$
\begin{aligned}
\dot{\Omega} & =\frac{2 \tilde{Q} \alpha^{2}}{\tau^{\mathrm{GR}}(\Omega)} \Omega \\
\dot{\alpha} & =-\left(\frac{1}{\tau^{\mathrm{GR}}}+\frac{1}{\tau^{\text {dis }}}\right) \alpha \\
C \dot{T} & =\frac{\tilde{J} M R^{2} \Omega}{\tau^{\text {dis }}} \alpha^{2}-L_{\mathrm{cool}}
\end{aligned}
$$


There we, following Owen et al. (1998), introduce dimensionless parameters

$$
\begin{aligned}
& \tilde{J}=\frac{1}{M R^{2 m}} \int_{0}^{R} \rho r^{2 m+2} \mathrm{~d} r \approx 1.64 \times 10^{-2} \\
& \tilde{I}=\frac{I}{M R^{2}}=\frac{8 \pi}{3 M R^{2}} \int_{0}^{R} \rho r^{4} \mathrm{~d} r \approx 0.261 \\
& \tilde{Q}=\frac{m(m+1) \tilde{J}}{4 \tilde{I}} \approx 9.4 \times 10^{-2}
\end{aligned}
$$

The numerical values are for $m=2 \mathrm{r}$-mode and Newtonian stellar model with polytropical EOS $P \propto \rho^{1+1 / n}$ with $n=1$. Note, in agreement with arguments by Levin \& Ushomirsky (2001b), the spin down rate given by Eq. (18) is directly associated with emission of gravitational waves.

The enhancement of the mode amplitude can be limited by nonlinear saturation (e.g., Bondarescu et al. 2007; Bondarescu \& Wasserman 2013; Haskell, Glampedakis, \& Andersson 2014), which can be described by substitution of the effective dissipation rate $\tau^{\text {dis }}=\left|\tau^{\mathrm{GR}}\right|$ instead of $\tau^{\text {dis }}$ into all equations (18)-(20).

\section{Comparison with previous works}

At first glance, the evolution equations (18-20) differ from equations derived by Owen et al. (1998); Ho \& Lai (2000) applied in vast majority of the papers dealing with the evolution of r-mode unstable NSs. Namely, in the leading order in mode amplitude the equations can be written in the form: ${ }^{6}$

$$
\begin{aligned}
\dot{\hat{\Omega}} & =\frac{2 \tilde{Q} \alpha^{2}}{\tau^{\mathrm{dis}}} \hat{\Omega} \\
\dot{\alpha} & =-\left(\frac{1}{\tau^{\mathrm{GR}}}+\frac{1}{\tau^{\mathrm{dis}}}\right) \alpha \\
C \dot{T} & =\frac{\tilde{J} M R^{2} \hat{\Omega}}{\tau^{\text {dis }}} \alpha^{2}-L_{\mathrm{cool}} .
\end{aligned}
$$

Here $\hat{\Omega}$ is the effective spin frequency, as introduced by Owen et al. (1998); Ho \& Lai (2000), which differs from $\Omega$, given by Eq. (1). Note, the rate of change of $\hat{\Omega}$ is associated with dissipation timescale, but not with instability timescale as in Eq. (18).

The reason of this difference originates from uncertainties in definition of mean spin frequency in CFS unstable neutron star arising from differential rotation, which can be generated in the star as a result of CFS instability (see, e.g., Spruit 1999; Rezzolla et al. 2000; Levin \& Ushomirsky 2001b; Friedman et al. 2016). Namely, Owen et al. (1998); Ho \& Lai (2000) assume that physical angular momentum associated with r-mode is equal to the canonical ${ }^{6}$ Here I neglect external torques and heating for the sake of sim-
plicity. angular momentum

$$
J_{\mathrm{c}}=-(3 / 2) \hat{\Omega} \tilde{J} M R^{2} \alpha^{2},
$$

and write total angular momentum as

$$
J=I \hat{\Omega}+J_{\mathrm{c}}
$$

As a result, the effective spin frequency is

$$
\hat{\Omega}=\left(1+\tilde{Q} \alpha^{2}\right) \Omega .
$$

After this change of variables our equations agree with results by Owen et al. (1998); Ho \& Lai (2000) in the leading order in $\alpha$.

Equations derived by Owen et al. (1998); Ho \& Lai (2000) also contain terms, which are of the next-order in $\alpha^{2}$. However I suppose that these terms (which do not agree for Owen et al. 1998 and Ho \& Lai 2000) include only part of the required next-order corrections. To be brief, their equations are based on the linear (leading order) perturbation theory and hence cannot predict next-to-leading order corrections accurately. For example, both Owen et al. (1998) and Ho \& Lai (2000) neglect corrections to the r-mode frequency associated with differential rotation (assuming r-mode frequency to be $\omega=4 \hat{\Omega} / 3$ in all orders in $\alpha$ ). However, as discussed by Chirenti, Skákala, \& Yoshida (2013), the differential rotation $\delta \Omega$ can affect the spin frequency at order of $\mathcal{O}(\delta \Omega / \Omega)$. As a result, the second order differential rotation, associated with excitation of r-modes (Spruit 1999; Rezzolla et al. 2000; Levin \& Ushomirsky 2001b; Friedman et al. 2016) leads to corrections to the r-mode frequency $\mathcal{O}\left(\alpha^{2}\right)$, and consequently to corrections of the same order for the energy pumping rate, given by Eq. (5). ${ }^{7}$ Note, the numerical value of these corrections depend on the differential rotation profile (Chirenti, Skákala, \& Yoshida 2013), which can be dramatically modified by the magnetic field in the star (see Chugunov 2015; Friedman et al. 2017, who also demonstrate that magnetic field windup by differential rotation do not suppress r-mode instability thanks to back reaction of magnetic field) or viscosity. To be accurate with the next order effects in the evolution equations, one needs additional modeling of the differential rotation profile. Fortunately, these effects should be negligible in any case, because current models of nonlinear saturation (e.g., Bondarescu et al. 2007; Bondarescu \& Wasserman 2013; Haskell, Glampedakis, \& Andersson 2014) predict low saturation amplitude $\alpha \lesssim 10^{-3}$ and actual observations constrain the r-mode amplitude in many

\footnotetext{
${ }^{7}$ In particular, to the best of my knowledge, there are no proofs that $\omega=4 \hat{\Omega} / 3$ is a better estimate for the $m=2 \mathrm{r}$-mode frequency than $\omega=4 \Omega / 3$, but these estimates differ at $\mathcal{O}\left(\alpha^{2}\right)$, see Eq. (29). The similar argument holds true for $\tau^{\mathrm{GR}}$ and $\tau^{\mathrm{dis}}$, which, according to Owen et al. (1998); Ho \& Lai (2000), should be calculated assuming uniform rotation frequency to be equal to $\hat{\Omega}$ (but not $\Omega$ ) in all orders in $\alpha$.
} 
potentially unstable neutrons stars even stronger (e.g., Mahmoodifar \& Strohmayer 2013; Alford \& Schwenzer 2015; Schwenzer et al. 2017; Chugunov et al. 2017). Thus I suggest to use leading order equations (18)-(20) (or equivalent equations 24-26) to model the evolution of r-mode unstable neutron stars and omit next-order terms.

\section{Summary and conclusions}

Using multipolar expansion of gravitational waves, formulated by Thorne (1980), I derive criterion of CFS instability for normal modes and evolution equations (5)(7) for a star, affected by this instability. The derivation does not appeal to the canonical energy formalism by Friedman \& Schutz (1978a,b). Eqs. (5)-(7) describe evolution of angular momentum, mode energy and temperature and can be applied for relativistic star. They are illustrated by r-mode instability in slowly rotating Newtonian stellar model (Eqs. 18- 20). In a latter case the evolution equations were earlier derived by Owen et al. (1998); Ho \& Lai (2000). At first glance they equations differ from those derived here. However, it is shown that it is spurious: in the leading order in mode amplitude the only difference is definition of the (effective) spin frequency, entering into the equations $(\Omega$ or $\hat{\Omega}$, see Eq. 29). Formulas, suggested by Owen et al. (1998); Ho \& Lai (2000) contain terms of the next order in the mode amplitude. However I argue that they do not include all of the required terms (as a result, these terms do not agree for Owen et al. 1998 and Ho \& Lai 2000) and thus it would be more self-consistent to restrict consideration to the leading order evolution equations (Eqs. 18- 20).

The difference in definition of the spin frequency $(\Omega$ and $\hat{\Omega}$, see Eq. 29) is associated with differential rotation, which can be generated during excitation of $\mathrm{r}$ modes by CFS instability (e.g., Spruit 1999; Ho \& Lai 2000; Rezzolla et al. 2000; Levin \& Ushomirsky 2001b; Friedman et al. 2016). As far as observed frequency is associated with specific point at the surface of neutron star (e.g. magnetic pole), the observed spin frequency $\Omega_{\text {obs }}$ can differ from both frequencies $\Omega$ and $\hat{\Omega}$. Important advantage of the formalism, suggested in this work, that it can be naturally extended to include effects of differential rotation (because it explicitly deals with second order axisymmetric velocity perturbations, see Sec. 3 ), and I plan to analyze them at the subsequent paper. As far as the spin down rate, given by Eq. (18), qualitatively agrees with arguments by Levin \& Ushomirsky (2001b): radiation of gravitation waves directly leads to spin down of the star (but not via dissipation of mode energy as it follows from Owen et al. 1998; Ho \& Lai 2000, see Eq. 24), I believe that $\Omega_{\text {obs }}$ would be closer to $\Omega$, than to $\hat{\Omega}$. However, this conclusion should be checked by accurate calculations

\section{Acknowledgements}

I'm grateful to Misha Gusakov and Elena Kantor for valuable comments and discussions. This study was supported by the Russian Science Foundation (Grant No. 14-12-00316).

\section{REFERENCES}

Alford, M. G. \& Schwenzer, K. 2014a, ApJ, 781, 26

—. 2014b, Physical Review Letters, 113, 251102

-. 2015, MNRAS, 446, 3631

Alpar, M. A., Pines, D., Anderson, P. W., \& Shaham, J. 1984, ApJ, 276, 325

Andersson, N. 1998, ApJ, 502, 708

Andersson, N., Glampedakis, K., \& Haskell, B. 2009, Phys. Rev. D, 79, 103009

Andersson, N. \& Kokkotas, K. D. 2001, International Journal of Modern Physics D, 10, 381

Andersson, N., Kokkotas, K. D., \& Stergioulas, N. 1999, ApJ, 516, 307

Beskin, V. S., Gurevich, A. V., \& Istomin, Y. N. 1993, Physics of the pulsar magnetosphere (Cambridge University Press)

Bildsten, L. 1998, ApJ, 501, L89

Bondarescu, R., Teukolsky, S. A., \& Wasserman, I. 2007, Phys. Rev. D, 76, 064019

Bondarescu, R. \& Wasserman, I. 2013, ApJ, 778, 9

Boyer, R. H. \& Lindquist, R. W. 1966, Physics Letters, 20, 504

Brown, E. F., Bildsten, L., \& Rutledge, R. E. 1998, ApJ, 504, L95

Chandrasekhar, S. 1970, Phys. Rev. Lett., 24, 611

Chirenti, C., Skákala, J., \& Yoshida, S. 2013, Phys. Rev. D, 87, 044043

Chugunov, A. I. 2015, MNRAS, 451, 2772

Chugunov, A. I., Gusakov, M. E., \& Kantor, E. M. 2014, MNRAS, 445, 385

Chugunov, A. I., Gusakov, M. E., \& Kantor, E. M. 2017, MNRAS, 468, 291

Friedman, J. L. 1978, Communications in Mathematical Physics, 62, 247

Friedman, J. L., Lindblom, L., \& Lockitch, K. H. 2016 , Phys. Rev. D, 93, 024023

Friedman, J. L., Lindblom, L., Rezzolla, L., \& Chugunov, A. I. 2017, Phys. Rev. D, submitted, eprint arXiv:1707.09419

Friedman, J. L. \& Morsink, S. M. 1998, ApJ, 502, 714

Friedman, J. L. \& Schutz, B. F. 1978a, ApJ, 221, 937

-. 1978b, ApJ, 222, 281

Friedman, J. L. \& Stergioulas, N. 2014, Instabilities of Relativistic Stars, ed. J. Bičák \& T. Ledvinka, 427

Ghosh, P. \& Lamb, F. K. 1979, ApJ, 234, 296

Glampedakis, K. \& Andersson, N. 2006a, Phys. Rev. D, 74,044040

-. 2006b, MNRAS, 371, 1311 
Gusakov, M. E., Chugunov, A. I., \& Kantor, E. M. 2014a, Phys. Rev. D, 90, 063001

—. 2014b, Phys. Rev. Lett., 112, 151101

Gusakov, M. E., Kaminker, A. D., Yakovlev, D. G., \& Gnedin, O. Y. 2005, MNRAS, 363, 555

Gusakov, M. E., Kantor, E. M., \& Reisenegger, A. 2015, MNRAS, 453, L36

Hartle, J. B. \& Sharp, D. H. 1967, ApJ, 147, 317

Haskell, B. 2015, International Journal of Modern Physics E, 24, 1541007

Haskell, B., Andersson, N., \& Passamonti, A. 2009, MNRAS, 397, 1464

Haskell, B., Degenaar, N., \& Ho, W. C. G. 2012, MNRAS, 424, 93

Haskell, B., Glampedakis, K., \& Andersson, N. 2014, MNRAS, 441, 1662

Ho, W. C. G. \& Lai, D. 2000, ApJ, 543, 386

Jackson, J. D. 1999, Classical electrodynamics, 3rd edn. (New York, NY: Wiley)

Jones, P. B. 2001, Physical Review Letters, 86, 1384

Kantor, E. M. \& Gusakov, M. E. 2017, MNRAS, 469, 3928

Kantor, E. M., Gusakov, M. E., \& Chugunov, A. I. 2016, MNRAS, 455, 739

Kastaun, W. 2011, Phys. Rev. D, 84, 124036

Kojima, Y. 1998, Monthly Notices of the Royal Astronomical Society, 293, 49

Kolomeitsev, E. E. \& Voskresensky, D. N. 2015, Phys. Rev. C, 91, 025805

Krüger, C., Gaertig, E., \& Kokkotas, K. D. 2010, Phys. Rev. D, 81, 084019

Lee, U. \& Yoshida, S. 2003, ApJ, 586, 403

Levin, Y. 1999, ApJ, 517, 328

Levin, Y. \& Ushomirsky, G. 2001a, MNRAS, 324, 917

-. 2001b, MNRAS, 322, 515

Lindblom, L. \& Owen, B. J. 2002, Phys. Rev. D, 65, 063006

Lindblom, L., Owen, B. J., \& Morsink, S. M. 1998, Phys. Rev. Lett., 80, 4843

Lockitch, K. H., Andersson, N., \& Friedman, J. L. 2001, Phys. Rev. D, 63, 024019

Lockitch, K. H., Andersson, N., \& Watts, A. L. 2004, Classical and Quantum Gravity, 21, 4661

Lockitch, K. H., Friedman, J. L., \& Andersson, N. 2003, Phys. Rev. D, 68, 124010

Mahmoodifar, S. \& Strohmayer, T. 2013, ApJ, 773, 140

McIntyre, M. E. 1981, Journal of Fluid Mechanics, 106, 331

Misner, C. W., Thorne, K. S., \& Wheeler, J. A. 1973, Gravitation (San Francisco: W.H. Freeman and Co.)

Nayyar, M. \& Owen, B. J. 2006, Phys. Rev. D, 73, 084001

Owen, B. J. 2010, Phys. Rev. D, 82, 104002

Owen, B. J., Lindblom, L., Cutler, C., Schutz, B. F., Vecchio, A., \& Andersson, N. 1998, Phys. Rev. D, 58,084020

pasa (2018)

doi:10.1017/pas.2018.xxx
Page, D., Lattimer, J. M., Prakash, M., \& Steiner, A. W. 2004, ApJS, 155, 623

Provost, J., Berthomieu, G., \& Rocca, A. 1981, A\&A, 94, 126

Reisenegger, A. 1995, ApJ, 442, 749

Rezzolla, L., Lamb, F. K., \& Shapiro, S. L. 2000, ApJ, 531, L139

Rieutord, M. 2001, ApJ, 550, 443

Ruoff, J. \& Kokkotas, K. D. 2002, MNRAS, 330, 1027

Sá, P. M. 2004, Phys. Rev. D, 69, 084001

Schwenzer, K., Boztepe, T., Güver, T., \& Vurgun, E. 2017, MNRAS, 466, 2560

Shternin, P. S., Baldo, M., \& Haensel, P. 2013, Phys. Rev. C, 88, 065803

Spruit, H. C. 1999, A\&A, 349, 189

Stergioulas, N. 2003, Living Reviews in Relativity, 6

Thorne, K. S. 1980, Reviews of Modern Physics, 52, 299

Varshalovich, D., Moskalev, A., \& Khersonskii, V. 1988, Quantum Theory of Angular Momentum (World Scientific Pub.)

Wang, Y.-M. 1995, ApJ, 449, L153

Yoshida, S. \& Lee, U. 2002, The Astrophysical Journal, 567, 1112

Yoshida, S. \& Lee, U. 2003a, MNRAS, 344, 207

—. 2003b, Phys. Rev. D, 67, 124019 